\title{
Tight-binding model investigation of the biaxial strain induced topological phase transition in $\mathrm{GeCH}_{3}$
}

\author{
Mohsen Rezaei, ${ }^{1}$ Esmaeil Taghizadeh Sisakht, ${ }^{1,2}$ Farhad Fazileh, ${ }^{1, *}$ Zahra Aslani, ${ }^{1}$ and F. M. Peeters ${ }^{2}$ \\ ${ }^{1}$ Department of Physics, Isfahan University of Technology, Isfahan 84156-83111, Iran \\ ${ }^{2}$ Department of Physics, University of Antwerp, Groenenborgerlaan 171, B-2020 Antwerpen, Belgium
}

(Received 5 June 2017; published 29 August 2017)

\begin{abstract}
We propose a tight-binding (TB) model, that includes spin-orbit coupling (SOC), to describe the electronic properties of methyl-substituted germanane $\left(\mathrm{GeCH}_{3}\right)$. This model gives an electronic spectrum in agreement with first principle results close to the Fermi level. Using the $\mathbb{Z}_{2}$ formalism, we show that a topological phase transition from a normal insulator (NI) to a quantum spin Hall (QSH) phase occurs at $11.6 \%$ biaxial tensile strain. The sensitivity of the electronic properties of this system on strain, in particular its transition to the topological insulating phase, makes it very attractive for applications in strain sensors and other microelectronic applications.
\end{abstract}

DOI: 10.1103/PhysRevB.96.085441

\section{INTRODUCTION}

Topological insulators (TIs) are a new state of matter that have attracted a lot of interest within the condensed matter physics community [1-6]. It is now well established that TIs are promising candidates for future advanced electronic devices. They possess a bulk insulating gap and conducting edge states. The edge states are protected by time-reversal symmetry (TRS) against backscattering and this property makes them robust against disorder and nonmagnetic defects. Consequently, the edge channels normally possess very high carrier mobility.

Among TI materials two-dimensional (2D) van der Waals systems have attracted a lot of attention during the past decade [7]. The interest in these systems originates from the discovery of graphene, which has a very high carrier mobility [200000 $\left.\mathrm{cm}^{2} /(\mathrm{V} \mathrm{s})\right]$, thermal conductivity, and mechanical strength [8,9]; however, its zero electronic band gap has severely limited its applicability in electronic devices. Also, the proposal for the existence of a topological insulating phase in graphene by Kane and Mele was shown to be unrealistic, because of its extremely small SOC strength [10,11]. Hence, extensive efforts have been devoted to open a band gap and increase the effective SOC in graphene or find other 2D systems with favorable SOC, carrier mobility, and appropriate band gap.

Other 2D materials such as single- or few-layer transition metal dichalcogenides (TMDs), boron nitride, silicene, germanene, phosphorene, stanene, and MXene, have been extensively explored [7,12-15].

Another important issue for applications in electronic industry is the compatibility of the material with current silicon-based electronic technology. Therefore, the group IV elements with honeycomb structure are more favorable for this purpose.

One method for tuning the electronic band structure of 2D systems is the use of surface functionalization. Functionalization of graphene with hydrogen, the so-called hydrogenterminated graphene or graphane, opens a sizable band gap,

*fazileh@cc.iut.ac.ir but its carrier mobility decreases dramatically to $10 \mathrm{~cm}^{2} /(\mathrm{V} \mathrm{s})$ [16]. Silicene and germanene the other analogs of graphene have also attracted much attention. However, the small band gap of these systems and mobility issues have limited their application for electronics. Functionalized germanene provide enhanced stability and tunable properties [17]. Compared with bulk Ge, surface functionalized germanene possess a direct and large band gap depending on the surface ligand. These materials can be synthesized via the topotactic deintercalation of layered Zintl phase precursors $[17,18]$. In contrast to TMDs, the weaker interlayer interaction allows for direct band gap single layer properties such as strong photoluminescence that are readily present without the need to exfoliate down to a single layer. Bianco et al. [19] produced experimentally hydrogen-terminated germanene, $\mathrm{GeH}$ (also called germanane). Recently the new material $\mathrm{GeCH}_{3}$ was synthesized [17], that exhibits an enhanced thermal stability. $\mathrm{GeCH}_{3}$ is thermally stable up to $250{ }^{\circ} \mathrm{C}$ which compares to $75^{\circ} \mathrm{C}$ for $\mathrm{GeH}$. The electronic structure of $\mathrm{GeCH}_{3}$ has been shown to be very sensitive to strain, which makes it very attractive for strain sensor applications [20-22]. It has also a high carrier mobility and pronounced light absorption which makes it attractive for light harvesting applications [21,22].

At present there exist already a few first-principle studies of $\mathrm{GeCH}_{3}$ that also include the effect of SOC [17,20-22]. To fully understand the physics behind the electronic band structure close to the Fermi level, we propose a TB model. Our TB model is fitted to the density functional theory (DFT) results both for the case with and without SOC. In the next part of this work we applied biaxial tensile strain to examine the effect of strain on the electronic properties of this system and compare our results with DFT calculations. The possibility of a topological phase transition in $\mathrm{GeCH}_{3}$ under biaxial tensile strain is also examined. Our finding that there is a transition to the QSH phase is further corroborated by the fact that we find TRS protected edge states in nanoribbons made out of $\mathrm{GeCH}_{3}$.

This paper is organized as follows. In Sec. II, we introduce the crystal structure and lattice constants of monolayer $\mathrm{GeCH}_{3}$. Our TB model with and without SOC is introduced in Sec. III, and the effect of strain on the electronic properties of monolayer $\mathrm{GeCH}_{3}$ is examined. In Sec. IV, using the $\mathbb{Z}_{2}$ formalism we demonstrate the existence of a topological phase 
(a)

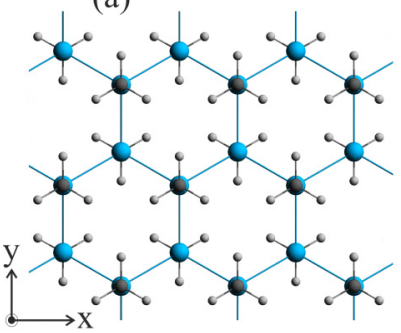

(c)

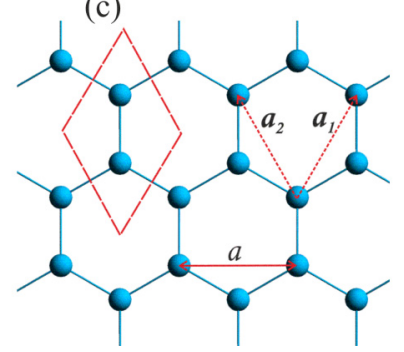

(b)

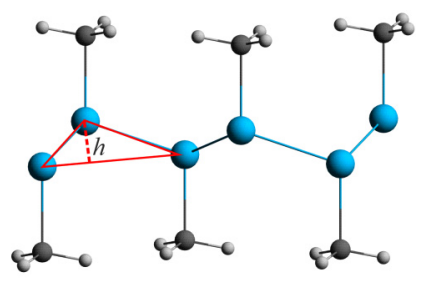

(d)

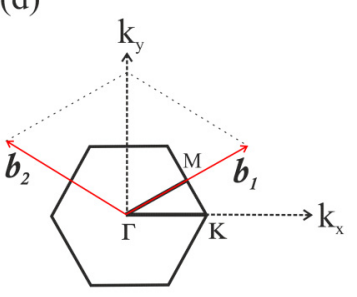

FIG. 1. Schematic top (a) and side (b) views of the monolayer $\mathrm{GeCH}_{3}$ structure. Blue, black, and gray balls indicate $\mathrm{Ge}, \mathrm{C}$, and $\mathrm{H}$ atoms, respectively. Ge atoms are sandwiched between two sheets of methyl groups. $h$ is the buckling of the structure. (c) Top view of the system eliminating the methyl group. $\boldsymbol{a}_{1}$ and $\boldsymbol{a}_{2}$ are the lattice vectors. (d) First Brillouin zone of the system with the reciprocal lattice vectors $\boldsymbol{b}_{1}$ and $\boldsymbol{b}_{2}$.

transition in the electronic properties of monolayer $\mathrm{GeCH}_{3}$ when biaxial tensile strain is applied. The paper is summarized in Sec.V.

\section{LATTICE STRUCTURE OF MONOLAYER GeCH}

The hexagonal atomic structure of monolayer $\mathrm{GeCH}_{3}$ and its geometrical parameters are shown in Figs. 1(a)-1(c). As shown in Figs. 1(a) and 1(b) it consists of three atomic layers where a buckled honeycomb sheet of Ge atoms is sandwiched between two outer methyl group layers. Each unit cell of monolayer $\mathrm{GeCH}_{3}$ consists of two Ge atoms and two $\mathrm{CH}_{3}$ groups. Previous DFT calculations gave for the lattice constant $a=3.954 \AA$, and the $\mathrm{Ge}-\mathrm{Ge}$ and $\mathrm{Ge}-\mathrm{C}$ bond lengths are $2.415 \AA$ and $1.972 \AA$, respectively [20]. The buckling height $h$, indicating the distance between two different Ge sublattices, is $0.788 \AA$.

We have chosen the $x$ and $y$ axes along the armchair and zigzag directions, respectively. The $z$ axis is in the normal direction to the plane of the monolayer $\mathrm{GeCH}_{3}$. With this definition of coordinates, the lattice vectors are written as $\boldsymbol{a}_{1}=a / 2(1, \sqrt{3}), \boldsymbol{a}_{2}=a / 2(-1, \sqrt{3})$, where the corresponding hexagonal Brillouin zone of the structure [see Fig. 1(d)] is determined by the reciprocal vectors $\boldsymbol{b}_{1}=$ $2 \pi / a(1, \sqrt{3} / 3), \boldsymbol{b}_{2}=2 \pi / a(-1, \sqrt{3} / 3)$.

\section{TIGHT-BINDING MODEL HAMILTONIAN}

Electronic structure of monolayer $\mathrm{GeCH}_{3}$ has been obtained by using DFT calculations in Ref. [20]. It is shown that the low-energy electronic properties of this system are dominated by $s, p_{x}$, and $p_{y}$ atomic orbitals of Ge atoms. DFT calculations including SOC interaction have shown that applying an

in-plane biaxial tensile strain induces a topological phase transition in the electronic properties of monolayer $\mathrm{GeCH}_{3}$ [20]. Although such a DFT approach provides valuable information regarding the electronic properties of such a system, it is limited to small computational unit cells. For example, large nanoribbons consisting of hundreds of atoms and including disorder require very large supercells which go beyond present day computational DFT capability. This motivated us to derive a TB model for monolayer $\mathrm{GeCH}_{3}$ that is sufficiently accurate to describe the low-energy spectrum and the electronic properties of this system.

In the following we will propose a low-energy TB model Hamiltonian that includes SOC for monolayer $\mathrm{GeCH}_{3}$. We show that our model is able to predict accurately the effect of strain on the electronic properties of the system.

\section{A. Model Hamiltonian without SOC}

We propose a TB model including $s, p_{x}$, and $p_{y}$ atomic orbitals with principal quantum number $n=4$ of Ge atoms to describe the low-energy spectrum of this system. The nearestneighbor effective TB Hamiltonian without SOC in the basis of $\left|s, p_{x}, p_{y}\right\rangle$ and in the second quantized representation is given by

$$
H_{0}=\sum_{i, \mu} E_{i \mu} c_{i \mu}^{\dagger} c_{i \mu}+\sum_{\langle i, j\rangle, \mu, v} t_{i \mu, j v}\left(c_{i \mu}^{\dagger} c_{j v}+\text { H.c. }\right),
$$

where $c_{i \mu}^{\dagger}$ and $c_{i \mu}$ represent the creation and annihilation operators for an electron in the $\mu$ th orbital of the $i$ th atom, $E_{i \mu}$ is the onsite energy of the $\mu$ th orbital of the $i$ th atom, and $t_{i \mu, j v}$ is the nearest-neighbor hopping amplitude between the $\mu$ th orbital of the $i$ th atom and $\nu$ th orbital of the $j$ th atom. We will show that this effective model is sufficiently accurate to describe the low-energy spectrum of this system.

Note that the above Hamiltonian is quite different from the effective Hamiltonian that describes the electronic properties of pristine germanene [23]. In the pristine honeycomb structures of the group IV elements, the effective low-energy spectrum is described by the outer $p_{z}$ atomic orbitals. However, in monolayer $\mathrm{GeCH}_{3}$, the $p_{z}$ orbitals mainly contribute to the $\sigma$ bonding between $\mathrm{Ge}$ and $\mathrm{C}$ atoms to form the energy bands that are far from the Fermi level. Therefore, we will neglect the contribution of the $p_{z}$ orbitals of the Ge atoms and the other orbitals of the $\mathrm{CH}_{3}$ molecule in our TB model.

With the above description, the hopping parameters of Eq. (1) can be expressed in terms of the standard Slater-Koster parameters as listed in the middle column of Table I, where $l=$ $\cos \theta \cos \phi_{0}$ and $m=\sin \theta \cos \phi_{0}$ are, respectively, functions of the cosine of the angles between the bond connecting two neighboring atoms with respect to $x$ and $y$ axes.

Using the Fourier transform of Eq. (1), and numerically diagonalizing the resulting Hamiltonian in $k$ space, one can fit to the $a b$ initio results in order to obtain the numerical values of the mentioned Slater-Koster parameters. The density functional calculation results [24] including the Heyd-ScuseriaErnzerhof (HSE) functional approximation [25] are used to parametrize the TB model given by Eq. (1). We have listed the obtained numerical values of these parameters in Table II. The numerically calculated TB energy bands of monolayer $\mathrm{GeCH}_{3}$ in the absence of strain, as shown in Fig. 2(a), are in excellent 
TABLE I. The nearest-neighbor hopping parameters between $s$ and $p$ orbitals are listed in the first column. The second column represents the hopping integrals as a function of the standard Slater-Koster parameters with direction-dependent quantities. The third column shows the nearest hopping parameters with the inclusion of applied strain.

\begin{tabular}{lcc}
\hline \hline Hopping parameters & Without strain & With biaxial strain \\
\hline$t_{s s}$ & $V_{s s \sigma}$ & $t_{s s}^{0}\left[1-2 \epsilon \cos \phi_{0}^{2}\right]$ \\
$t_{s p_{x}}$ & $l V_{s p \sigma}$ & $t_{s p_{x}}^{0}\left[1-2 \epsilon \cos \phi_{0}^{2}+\eta \epsilon \tan \phi_{0}\right]$ \\
$t_{s p_{y}}$ & $m V_{s p \sigma}$ & $t_{s p_{y}}^{0}\left[1-2 \epsilon \cos \phi_{0}^{2}+\eta \epsilon \tan \phi_{0}\right]$ \\
$t_{p_{x} p_{x}}$ & $l^{2} V_{p p \sigma}+\left(1-l^{2}\right) V_{p p \pi}$ & $t_{p_{x} p_{x}}^{0}\left[1-2 \epsilon \cos \phi_{0}^{2}+2 \eta \epsilon \tan \phi_{0}\right]-2 \eta \epsilon \tan \phi_{0} V_{p p \pi}$ \\
$t_{p_{y} p_{y}}$ & $m^{2} V_{p p \sigma}+\left(1-m^{2}\right) V_{p p \pi}$ & $t_{p_{y} p_{y}}^{0}\left[1-2 \epsilon \cos \phi_{0}^{2}+2 \eta \epsilon \tan \phi_{0}\right]-2 \eta \epsilon \tan \phi_{0} V_{p p \pi}$ \\
$t_{p_{x} p_{y}}$ & $\operatorname{lm}\left(V_{p p \sigma}-V_{p p \pi)}\right.$ & $t_{p_{x} p_{y}}^{0}\left[1-2 \epsilon \cos \phi_{0}^{2}+2 \eta \epsilon \tan \phi_{0}\right]$ \\
\hline \hline
\end{tabular}

agreement with the $a b$ initio results. The direct band gap of monolayer $\mathrm{GeCH}_{3}$ at the $\Gamma$ point is $1.82 \mathrm{eV}$.

\section{B. Strain effects}

Applying strain to a system modifies its electronic properties [26]. This is due to the fact that it changes both the bond lengths and bond angles leading to a modulation of the hopping parameters that determine the electronic properties of the system.

An accurate prediction of the electronic properties of the system in the presence of different types of strain, is a stringent test of the accuracy of our TB model. To this end, we now first calculate the modification of the hopping parameters when biaxial tensile strain is applied to the plane of monolayer $\mathrm{GeCH}_{3}$. Then we will study the modification of the energy spectrum in the presence of such a strain to show that our results agree very well with the DFT calculations. This particular type of strain noticeably simplifies our calculations. When biaxial tensile strain is applied in the plane of monolayer $\mathrm{GeCH}_{3}$ leaves the honeycomb nature of its lattice intact and the initial lattice vectors $\boldsymbol{a}_{1}^{0}$ and $\boldsymbol{a}_{2}^{0}$ evolve to the deformed ones $\boldsymbol{a}_{1}$ and $\boldsymbol{a}_{2}$. Therefore, the vector $\boldsymbol{r}_{0}=\left(x_{0}, y_{0}, z_{0}\right)$, in the presence of in-plane strain is deformed into $\boldsymbol{r}=(x, y, z)=\left[\left(1+\epsilon_{x}\right) x_{0},\left(1+\epsilon_{y}\right) y_{0}, z_{0}\right]$, where $\epsilon_{x}$ and $\epsilon_{y}$ are the strain in the direction of the $x$ and $y$ axes, respectively. In the following, for simplicity we assume that the strengths of the applied biaxial strains in the two directions are equal, i.e., $\epsilon_{x}=\epsilon_{y}=\epsilon$. In the linear deformation regime, one can perform an expansion of the norm of $r$ to first order in $\epsilon_{x}$ and $\epsilon_{y}$ which results in

$$
r \simeq\left(1+\alpha_{x} \epsilon_{x}+\alpha_{y} \epsilon_{y}\right) r_{0}=\left[1+\left(\alpha_{x}+\alpha_{y}\right) \epsilon\right] r_{0},
$$

where $\alpha_{x}=\left(x_{0} / r_{0}\right)^{2}$ and $\alpha_{y}=\left(y_{0} / r_{0}\right)^{2}$ are coefficients related to the geometrical structure of $\mathrm{GeCH}_{3}$. For the three nearest neighbor Ge atoms, one can write $\alpha_{x}+\alpha_{y}=\cos ^{2} \phi_{0}$, where $\phi_{0}$ is the initial buckling angle. We note that in the presence of biaxial strain, the bond lengths and buckling angles are both

TABLE II. The values of the Slater-Koster parameters in units of $\mathrm{eV}$ as obtained from a fitting to the ab-initio results.

\begin{tabular}{cccccc}
\hline \hline$V_{s s \sigma}$ & $V_{s p \sigma}$ & $V_{p p \sigma}$ & $V_{p p \pi}$ & $E_{s}$ & $E_{p}$ \\
\hline-2.20 & 2.62 & 2.85 & -0.85 & -5.09 & 2.1 \\
\hline
\end{tabular}

altered. Thus, we consider their effects on the modification of the hopping parameters, simultaneously. Based on elasticity theory, we know that the main features of the mechanical properties in a covalent material are determined by the structure of the system and the strength of the covalent bonds. Therefore, one can expect that the change of the buckling angle in germanene [23] and $\mathrm{GeCH}_{3}$ be akin. The variation of the buckling angle [23] as a function of biaxial strain can be fit to the linear form $\phi=\phi_{0}-\eta \epsilon$ (see Fig. 3), where $\eta=-30$.
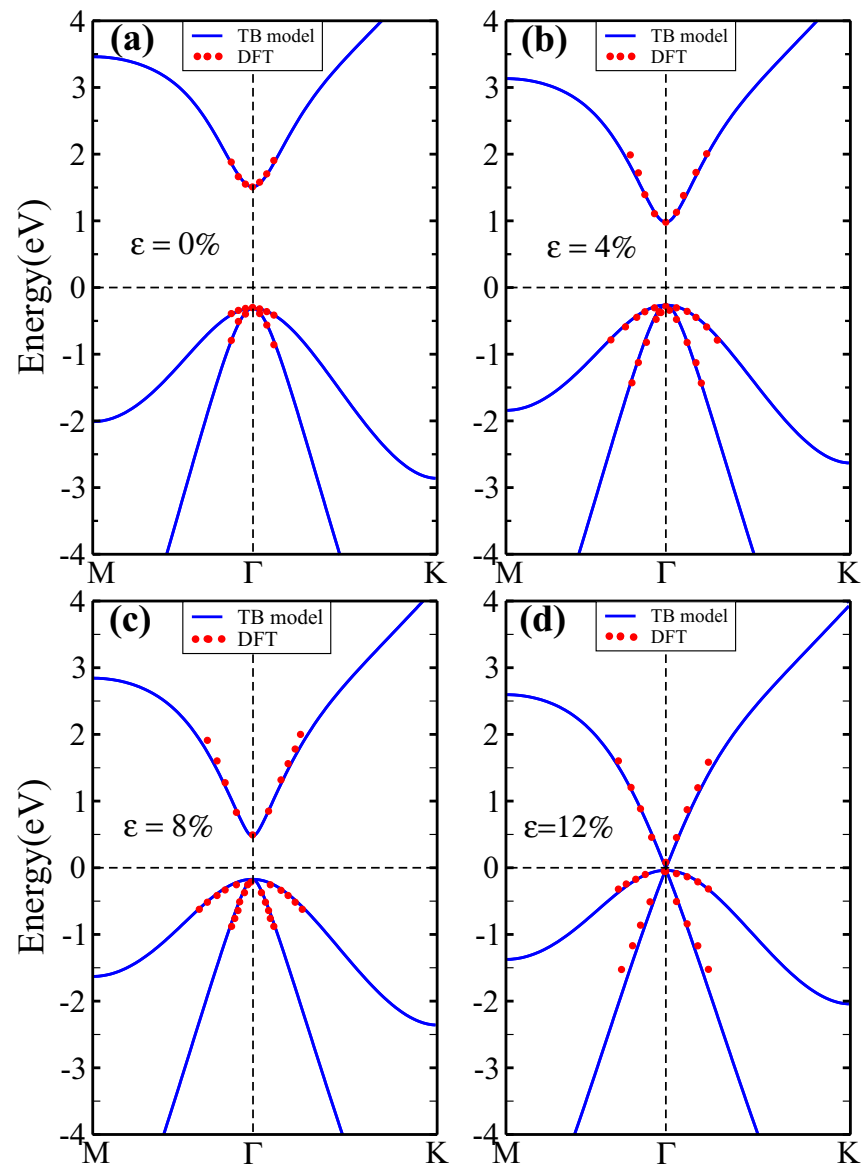

FIG. 2. The TB band structure of the $\mathrm{GeCH}_{3}$ isolated monolayer without SOC in the presence of (a) $0 \%$, (b) $4 \%$, (c) $8 \%$, and (d) $12 \%$ biaxial tensile strain. Symbols represent the HSE data taken from [24]. 


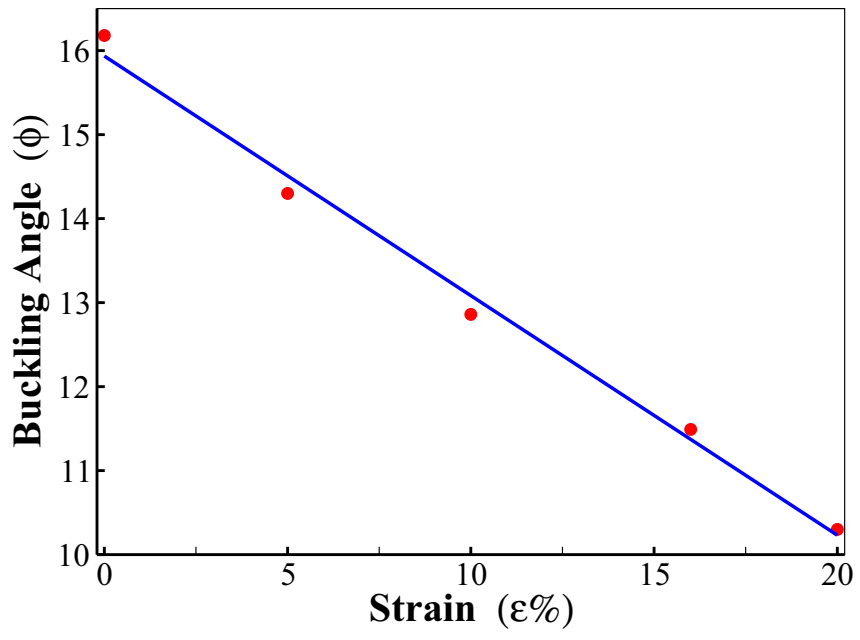

FIG. 3. Variation of the buckling angle as a function of biaxial tensile strain. Symbols represent the DFT data for germanene [23] and the solid line is the fit to this data.

According to the Harrison rule [27], the standard SlaterKoster parameters related to $s$ and $p$ orbitals are proportional to the bond length $r$ as $V_{\alpha \beta \gamma} \propto 1 / r^{2}$. Using Eq. (2), the modified parameters are given by

$$
V_{\alpha \beta \gamma}=\left(1-2 \epsilon \cos ^{2} \phi_{0}\right) V_{\alpha \beta \gamma}^{0} .
$$

One can then use the change of the buckling angle and the Slater-Koster parameters to obtain the modified hopping parameters as listed in the last column of Table I, where $t_{\alpha \beta}^{0}$ represents the unstrained hopping parameters. For instance, the new hopping parameter $t_{s p_{x}}$ can be approximated by

$$
\begin{aligned}
t_{s p_{x}} & =t_{s p_{x}}^{0}+\left(\frac{\partial t_{s p_{x}}}{\partial r}\right)_{r_{0}} \Delta r+\left(\frac{\partial t_{s p_{x}}}{\partial \phi}\right)_{\phi_{0}} \Delta \phi \\
& =t_{s p_{x}}^{0}-2 \cos \theta \cos \phi_{0} V_{s p \sigma}^{0} \frac{\Delta r}{r_{0}}-\cos \theta \sin \phi_{0} V_{s p \sigma}^{0} \Delta \phi .
\end{aligned}
$$

Substituting $\Delta r / r_{0}=\epsilon \cos ^{2} \phi_{0}$ and $\Delta \phi=-\eta \epsilon$ into the above equation gives

$$
t_{s p_{x}}=t_{s p_{x}}^{0}\left[1-\epsilon\left(2 \cos ^{2} \phi_{0}-\eta \tan \phi_{0}\right)\right] .
$$

In a similar way, one can obtain the other modified hopping parameters in order to study the evolution of the energy spectrum of monolayer $\mathrm{GeCH}_{3}$ as a function of applied biaxial tensile strain.

Straightforward substitution of the new hopping parameters in the Hamiltonian, Eq. (1), gives the Hamiltonian for the strained system. The calculated TB energy spectrum in the presence of biaxial tensile strain with strengths of $4 \%, 8 \%$, and $12 \%$ are shown in Figs. 2(b), 2(c) and 2(d), which are in excellent agreement with the DFT results [20,24]. We show in Fig. 4 the dependence of the band gap of $\mathrm{GeCH}_{3}$ as a function of biaxial tensile strain. Notice the good agreement between both DFT and TB approaches demonstrating the validity of our proposed TB model.

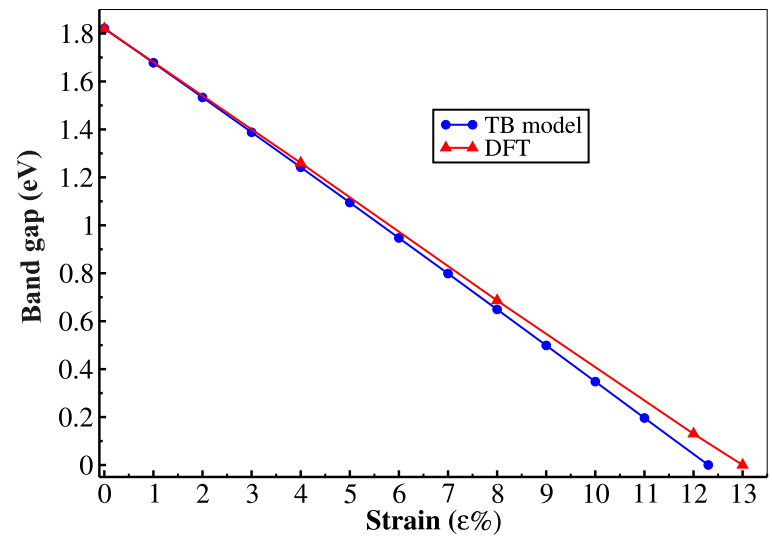

FIG. 4. Comparison of the variation of energy band gap vs biaxial strain between TB model and HSE calculations [20].

\section{Spin-orbit coupling}

Spin-orbit interaction is a relativistic correction to the Schrödinger equation. It can significantly affect the electronic properties of systems that consist of heavier elements. In such systems, the major part of SOC originates from the orbital motion of electrons close to the atomic nuclei. In the Slater-Koster approximation, one can assume an effective spherical atomic potential $V_{i}(\boldsymbol{r})$, at least in the region near the nucleus. Therefore, one can substitute $\nabla V_{i}(\boldsymbol{r})=\left(d V_{i} / d r\right) \boldsymbol{r} / r$ and $s=\hbar / 2 \sigma$ into the general form for the SOC term [28,29],

$$
H_{\mathrm{SOC}}=-\frac{\hbar}{4 m_{0}^{2} c^{2}}(\nabla V \times \boldsymbol{p}) \cdot \boldsymbol{\sigma},
$$

to obtain the SOC in the form of

$$
H_{\mathrm{SOC}}=\lambda(r) \boldsymbol{L} \cdot \boldsymbol{\sigma},
$$

where $\lambda(r)=1 / 2 m_{0}^{2} c^{2} r(d V / d r)$ is a radial function whose value depends on the type of atomic species. In the above equations, $\hbar, m_{0}, c$, and $\boldsymbol{p}$, are Plank constant, free mass of electron, speed of light, and momentum, respectively; and $\boldsymbol{\sigma}, \boldsymbol{L}$, and $\boldsymbol{s}$ represent the Pauli matrices, angular momentum operator, and electron spin operator, respectively.

Using the well-known ladder operators $L_{ \pm}$and $S_{ \pm}$, one can obtain the matrix representation of the SOC Hamiltonian in the basis set of $|\uparrow, \downarrow\rangle \otimes\left|s_{1}, p_{x 1}, p_{y 1}, s_{2}, p_{x 2}, p_{y 2}\right\rangle$ for monolayer $\mathrm{GeCH}_{3}$ with matrix elements,

$$
\left\langle\alpha_{i}\left|H_{\mathrm{SOC}}\right| \beta_{i}\right\rangle=\lambda_{i}\langle\boldsymbol{L} \cdot \boldsymbol{\sigma}\rangle_{\alpha \beta},
$$

where $\alpha_{i}$ and $\beta_{i}$ represent the atomic orbitals of the $i$ th atom. Note that since the two atom bases in the unit cell of the monolayer $\mathrm{GeCH}_{3}$ are the same, we have $\lambda_{1}=\lambda_{2}=\lambda$. There is no matrix element between $s$ and $p$ orbitals for the SOC interaction. Therefore, the resulting Hamiltonian can be written in the form,

$$
\begin{aligned}
H= & \sum_{i, \mu, \sigma} E_{i \mu} c_{i \mu \sigma}^{\dagger} c_{i \mu \sigma}+\sum_{\langle i, j\rangle, \mu, \nu, \sigma} t_{i \mu, j v}\left(c_{i \mu \sigma}^{\dagger} c_{j \nu \sigma}+\text { H.c. }\right) \\
& +\lambda \sum_{i, \alpha, \beta, \gamma, \sigma, \sigma^{\prime}} c_{i p_{\alpha} \sigma}^{\dagger} c_{i p_{\beta} \sigma^{\prime}}\left(-i \epsilon_{\alpha \beta \gamma} \sigma_{\sigma \sigma^{\prime}}^{\gamma}\right)
\end{aligned}
$$




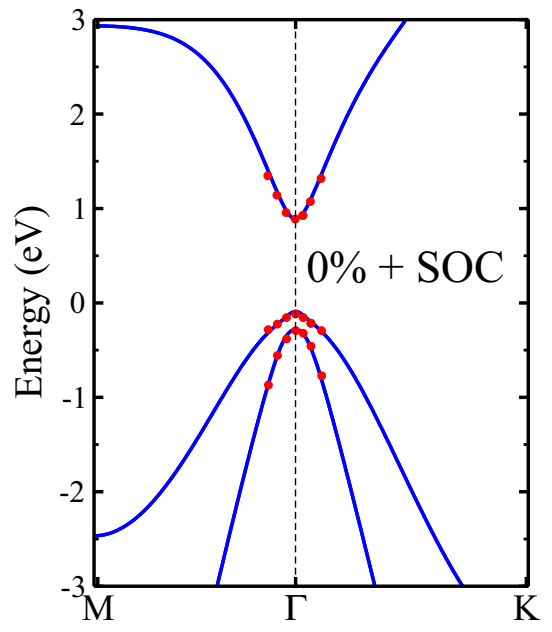

FIG. 5. The multiorbital TB spectrum of the $\mathrm{GeCH}_{3}$ monolayer with SOC. Symbols represent the LDA data taken from [20].

where $\mu$ and $\nu$ are either $s, p_{x}$, or $p_{y}$ orbitals; $\alpha$ and $\beta$ run over $x, y$, and $z$; and $\epsilon$ is the Levi-Civita symbol. $\sigma_{\sigma \sigma^{\prime}}^{\gamma}$ are Pauli matrices.

The value of the strength $\lambda$ of the SOC should be chosen either in agreement with experiment or by fitting the TB bands to the $a b$ initio results near some $k$ points such that it gives the correct band gap. In order to evaluate the strength of the SOC for $\mathrm{Ge}$ atoms in monolayer $\mathrm{GeCH}_{3}$, we fitted the spectrum obtained from our multiorbital TB model to the one from density functional calculations within the local density approximation (LDA) for the exchange correlation in Ref. [20]. As shown in Fig. 5, there is excellent agreement between the TB spectrum and the DFT results for the SOC strength $\lambda=0.096 \mathrm{eV}$. We adopt this SOC strength in the following calculations of the TB spectrum when we use the hopping parameters from Table II.

The TB energy spectrum of monolayer $\mathrm{GeCH}_{3}$ are shown in Figs. 6(a) and 6(b) for $0 \%$ and $12.5 \%$ strain, respectively. Note that due to the presence of time reversal and inversion
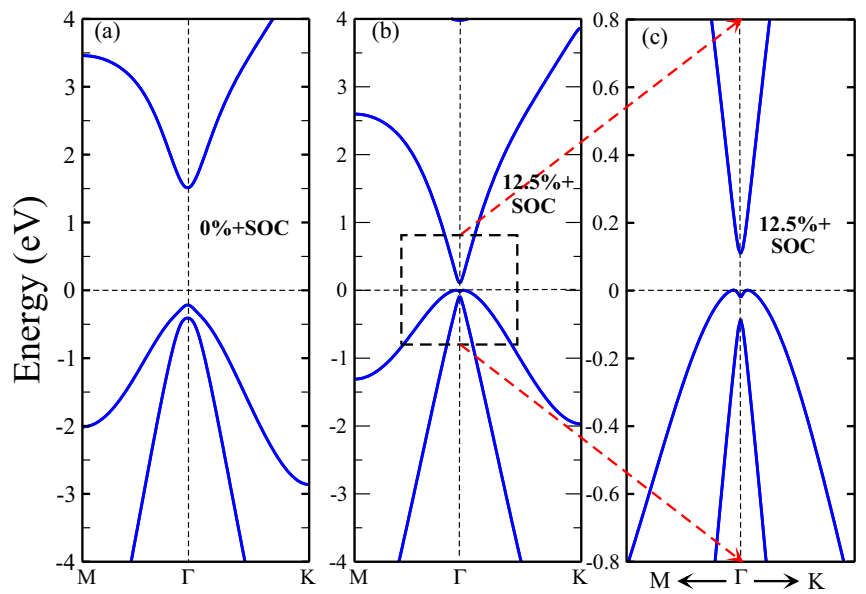

FIG. 6. The TB band structure of $\mathrm{GeCH}_{3}$ isolated monolayer with SOC in the presence of (a) $0 \%$, and (b) $12.5 \%$ biaxial tensile strain. (c) Zoomed-in view of (b).

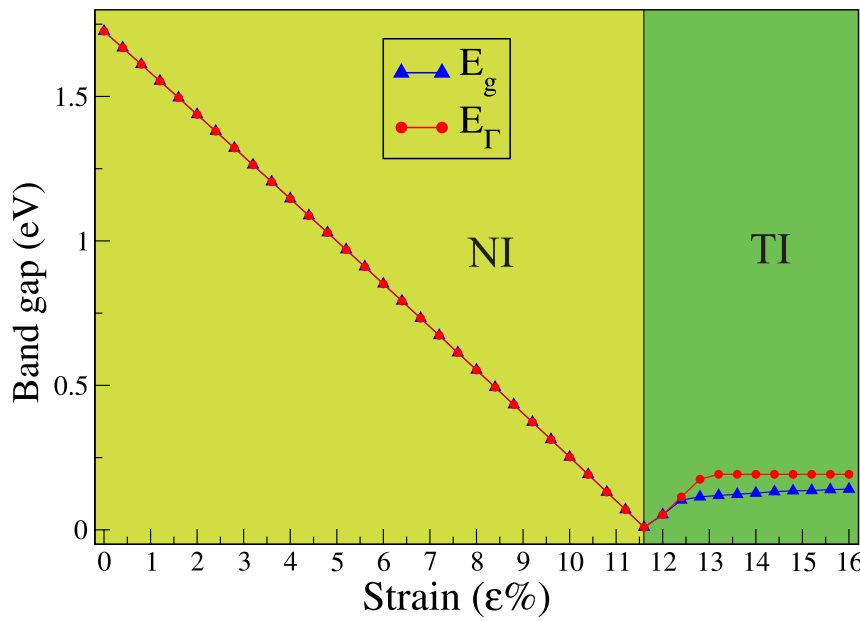

FIG. 7. The calculated band gaps of monolayer $\mathrm{GeCH}_{3}$ as a function of biaxial strain at the $\Gamma$ point $E_{\Gamma}$, and the global gap $E_{g}$. The two distinct colored regions show the different trivial and band inverted phases.

symmetry, each band in the energy spectrum of monolayer $\mathrm{GeCH}_{3}$ is doubly degenerate. As shown in Fig. 7, by applying biaxial tensile strain, the global band gap located at $\Gamma$ gradually decreases and eventually a band inversion occurs at $11.6 \%$ strain. By further increasing strain, the induced band gap due to SOC [see Figs. 6(b), and 6(c)] becomes indirect, and at a reasonable strength of $12.8 \%$ reaches the value of $115 \mathrm{meV}$.

One can use the TB spectrum of Fig. 6 to calculate the effective masses of electrons and holes near the conduction band minimum (CBM) and the valence band maximum (VBM). The results, in unit of free electron mass $m_{0}$, are listed in Table III for $0 \%, 6 \%, 9 \%$, and $12.5 \%$ biaxial tensile strain. Note that the electron and hole effective masses near the CBM and VBM along the two directions of $\Gamma-\mathrm{K}$ and $\Gamma-\mathrm{M}$ are the same.

Another way to test the validity of our TB model is its ability to predict a possible topological phase transition in the electronic properties of monolayer $\mathrm{GeCH}_{3}$. In the next section we will study the strain-induced topological phase in monolayer $\mathrm{GeCH}_{3}$ using our TB model.

\section{TOPOLOGICAL PHASE TRANSITION

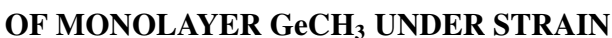

In the previous section, using the TB model including SOC, we showed that the monolayer $\mathrm{GeCH}_{3}$ is a NI. We also

TABLE III. The effective mass of electron and hole near the CBM and VBM in unit of free electron mass $m_{0}$. The electron and hole effective masses along the two directions of $\Gamma-\mathrm{K}$ and $\Gamma-\mathrm{M}$ are the same.

\begin{tabular}{lcc}
\hline \hline Strain $(\epsilon) \backslash$ Effective mass $\left(m / m_{0}\right)$ & Electron & Hole \\
\hline $0 \%$ & 0.135 & 0.157 \\
$6 \%$ & 0.074 & 0.105 \\
$9 \%$ & 0.045 & 0.058 \\
$12.5 \%$ & 0.033 & 0.316 \\
\hline \hline
\end{tabular}


showed that one can manipulate its electronic properties by applying in-plane biaxial strain. It is clear from Eq. (8) that SOC preserves the TRS. Thus, the monolayer $\mathrm{GeCH}_{3}$ can exhibit a QSH phase when its energy spectrum is manipulated by an external parameter that does not break TRS. The $\mathbb{Z}_{2}$ classification is a well-known approach to distinguish between the two different NI and TI phases [1,2]. In the following, we briefly introduce the lattice version of the Fu-Kane formula [30] to calculate the $\mathbb{Z}_{2}$ invariant. Then, we show numerically that by applying biaxial tensile strain, a change in the bulk topology of the monolayer $\mathrm{GeCH}_{3}$ occurs.

\section{A. Calculation of the $\mathbb{Z}_{2}$ invariant}

The Fu-Kane formula [30] for the calculation of the $\mathbb{Z}_{2}$ invariant is given by

$$
\left.\mathbb{Z}_{2}=\frac{1}{2 \pi i}\left[\oint_{\partial \mathrm{HBZ}} d \boldsymbol{k} \cdot \mathcal{A}(\boldsymbol{k})-\int_{\mathrm{HBZ}} d^{2} k \mathcal{F}(\boldsymbol{k})\right]\right](\bmod 2),
$$

where the integral is taken over half the Brillouin zone as denoted by HBZ. Here, the Berry gauge potential $\mathcal{A}(\boldsymbol{k})$ and the Berry field strength $\mathcal{F}(\boldsymbol{k})$ are given by $\sum_{n}\left\langle u_{n}(\boldsymbol{k}) \mid \nabla_{n} u_{n}(\boldsymbol{k})\right\rangle$ and $\nabla_{\boldsymbol{k}} \times\left.\mathcal{A}(\boldsymbol{k})\right|_{z}$, respectively; $u_{n}(\boldsymbol{k})$ represents the periodic part of the Bloch wave function with band index $n$, and the summation in $\mathcal{A}(\boldsymbol{k})$ runs over all occupied states.

Note that, in this approach, one has to do some gauge fixing procedure [31] to fulfill the TRS constraints and the periodicity of the $k$ points which are related by a reciprocal lattice vector $\boldsymbol{G}$. Moreover, due to the TRS and the inversion symmetry in the monolayer $\mathrm{GeCH}_{3}$, each band is at least doubly degenerate. Therefore, one needs to generalize the definition of $\mathcal{A}$ and $\mathcal{F}$ to non-Abelian gauge field analogies [32] constructed from the 2M-dimensional ground-state multiplet $|\psi(k)\rangle=\left(\left|u_{1}(k)\right\rangle, \ldots,\left|u_{2 M}(k)\right\rangle\right)$, associated with the Hamiltonian $\mathcal{H}(k)\left|u_{n}(k)\right\rangle=E_{n}(k)\left|u_{n}(k)\right\rangle$ [31,32].

In order to compute the $\mathbb{Z}_{2}$ invariant, a lattice version of Eq. (10) is more favorable for numerical calculations. To this end, one can simply convert the equivalent rhombus shape of the honeycomb Brillouin zone in $k$ space as shown in Figs. 8(a) and 8(b), into a unit square in $q$ space by the following change of variables:

$$
k_{x}=\frac{2 \pi}{a}\left(q_{x}-q_{y}\right), \quad k_{y}=\frac{2 \pi}{\sqrt{3} a}\left(q_{x}+q_{y}\right) .
$$
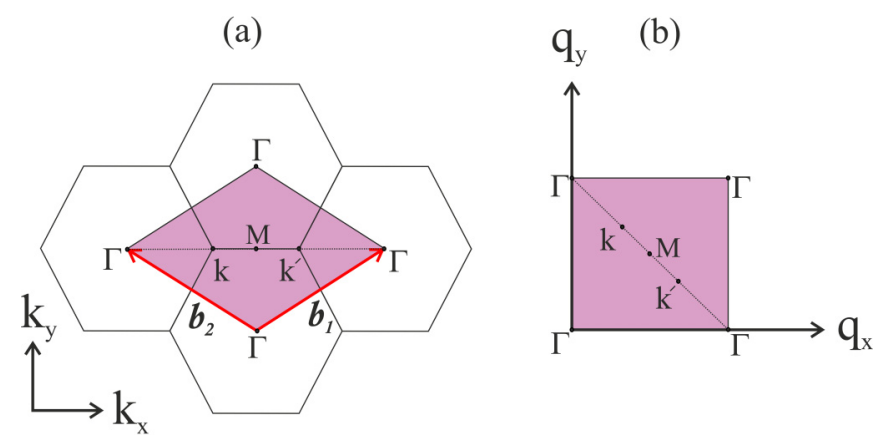

FIG. 8. Conversion of the equivalent (a) rhombus shape of the honeycomb Brillouin zone in $k$ space into a (b) unit square in $q$ space.
This allows us to use the more simple lattice version of Eq. (10) [31],

$$
\mathbb{Z}_{2}=\frac{1}{2 \pi i}\left[\sum_{q_{l} \in \partial \mathrm{HBZ}} A_{x}\left(q_{l}\right)-\sum_{q_{l} \in \mathrm{HBZ}} F_{x y}\left(q_{l}\right)\right](\bmod 2),
$$

where the lattice sites of the Brillouin zone are labeled by $q_{l}$. Thus the above-mentioned gauge fixing procedure and TRS constraints are applied on the equivalent $q$ points. Using the so-called unimodular link variable [31],

$$
U_{\hat{\mu}}\left(q_{l}\right)=\frac{\operatorname{det} \psi^{\dagger}\left(q_{l}\right) \psi\left(q_{l}+\hat{\mu}\right)}{\left|\operatorname{det} \psi^{\dagger}\left(q_{l}\right) \psi\left(q_{l}+\hat{\mu}\right)\right|},
$$

where $\hat{\mu}$ denotes a unit vector in the $q_{x}-q_{y}$ plane, one can define the Berry potential and Berry field in Eq. (12) as

$$
\begin{gathered}
A_{x}\left(q_{l}\right)=\ln U_{x}\left(q_{l}\right), \\
F_{x y}\left(q_{l}\right)=\ln \frac{U_{x}\left(q_{l}\right) U_{y}\left(q_{l}+\hat{x}\right)}{U_{y}\left(q_{l}\right) U_{x}\left(q_{l}+\hat{y}\right)} .
\end{gathered}
$$

Note that both the Berry potential and the Berry field strength are defined within the branch of $A_{x}\left(q_{l}\right) / i \in(-\pi, \pi)$ and $F_{x y}\left(q_{l}\right) / i \in(-\pi, \pi)$.

The numerical results of the $\mathbb{Z}_{2}$ invariant are shown in Fig. 9. As seen, for $\epsilon<11.6 \%$, the monolayer $\mathrm{GeCH}_{3}$ is an NI and at the critical value of $\epsilon=11.6 \%$, the $\mathbb{Z}_{2}$ invariant jumps from 0 to 1 , indicating a strain-induced TI phase transition in the electronic properties of the system. The topologically protected global bulk gap for a strain of $12.8 \%$ is $115 \mathrm{meV}$, which is much larger than the thermal energy at room temperature and therefore the monolayer $\mathrm{GeCH}_{3}$ is an excellent candidate for strain related applications.

In the next subsection we examine the formation of topologically protected edge states in a typical nanoribbon with zigzag edges when the system is driven into the TI phase by applying biaxial tensile strain.

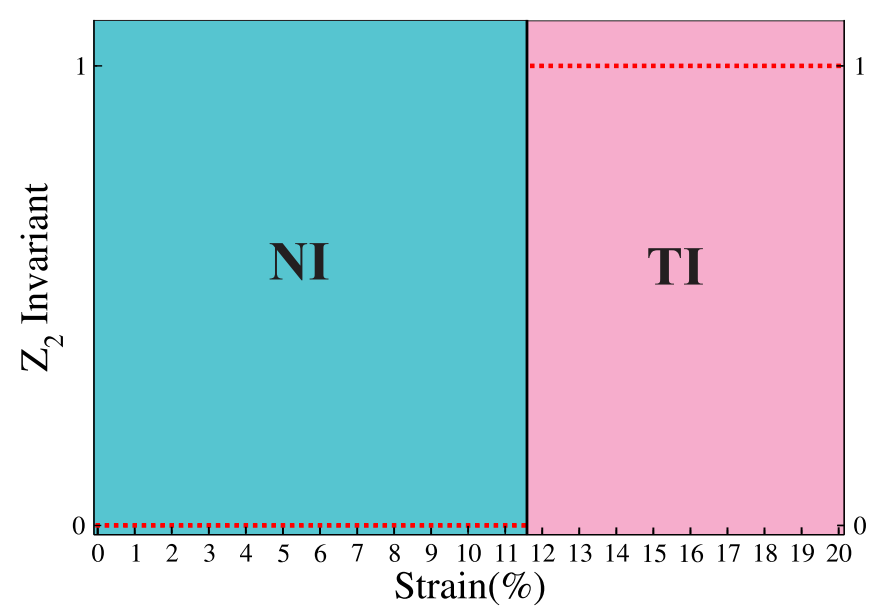

FIG. 9. Calculation of the $\mathbb{Z}_{2}$ invariant for the monolayer $\mathrm{GeCH}_{3}$ in the presence of biaxial strain. The two NI and TI phases are represented by regions of different colors and delimited by a black line at the critical value of $11.6 \%$. 


\section{B. Electronic properties of $\mathrm{GeCH}_{3}$ nanoribbons under strain}

The appearance of helical gapless states at the edge of a 2D topological insulator is a crucial consequence of its nontrivial bulk topology. In the previous section, we showed that a jump from 0 to 1 in the $\mathbb{Z}_{2}$ invariant for biaxial strain at $\epsilon>11.6 \%$ takes place, demonstrating a topological phase transition in the electronic properties of the monolayer $\mathrm{GeCH}_{3}$. As an example, in this subsection, we study the 1D energy bands of $\mathrm{GeCH}_{3}$ nanoribbons with zigzag edges in the presence of biaxial tensile strain. Our TB model predicts the appearance of topologically protected edge states with increasing strain when the $\mathbb{Z}_{2}$ invariant becomes 1 . We denote the width of the zigzag $\mathrm{GeCH}_{3}$ nanoribbon ( $\mathrm{z}-\mathrm{GeCH}_{3}-\mathrm{NR}$ ) by $\mathrm{N}$, which is the number of zigzag chains across the ribbon width. To calculate the energy spectrum of a $\mathrm{z}-\mathrm{GeCH}_{3}-\mathrm{NR}$ with width $\mathrm{N}$, we construct its supercell Hamiltonian $\left(H^{\mathrm{SC}}\right)$ in the basis of $|\psi\rangle \equiv|\uparrow, \downarrow\rangle \otimes$ $\left|s_{H_{0}}, s_{1}, p_{x 1}, p_{y 1}, \ldots, s_{2 N}, p_{x 2 N}, p_{y 2 N}, s_{H_{1}}\right\rangle$ where $s_{i}, p_{x i}$, and $p_{y i}$ represent the $s, p_{x}$, and $p_{y}$ orbitals of Ge atoms along the nanoribbon width. $\left|s_{H_{0}}\right\rangle$ and $\left|s_{H_{1}}\right\rangle$ represent the atomic orbitals of $\mathrm{H}$ atoms that are introduced to passivate the $\mathrm{Ge}$ atoms on each edge, respectively. We assume that the width of the nanoribbon is large enough that the interaction between the two edges is negligible, and one can safely neglect the tiny change of the hopping parameters due to the passivation procedure. Therefore, one can write the matrix elements of the nanoribbon Hamiltonian $H^{\mathrm{SC}}=H_{0}^{\mathrm{SC}}+H_{\mathrm{SOC}}^{\mathrm{SC}}$ as

$$
\begin{aligned}
M_{i \mu, j v}^{\sigma \sigma^{\prime}}= & \left\langle\psi\left|H^{\mathrm{SC}}\right| \psi\right\rangle_{i \mu, j v}^{\sigma \sigma^{\prime}} \\
= & E_{i \mu} \delta_{i j} \delta_{\mu \nu} \delta_{\sigma \sigma^{\prime}} \\
& +\delta_{\sigma \sigma^{\prime}} \sum_{n} t_{i \mu, j v} e^{i \boldsymbol{k} \cdot \boldsymbol{R}_{0 n}}+\lambda_{i} \delta_{i j}\langle\boldsymbol{L} \cdot \boldsymbol{\sigma}\rangle_{\mu \nu}^{\sigma \sigma^{\prime}},
\end{aligned}
$$

where $i, j$ are the basis site indices in a supercell; $\mu, v$ denote the atomic orbitals; $\sigma, \sigma^{\prime}$ denote the spin degrees of freedom; and $\boldsymbol{R}_{0 n}$ is the translational vector of the $n$th supercell. The corresponding onsite energy of Ge atoms and the hopping parameters pertinent to the Ge-Ge bonds are substituted from Tables I and II. Moreover, one has to define the onsite energy $E_{H}^{s}$, and the hopping parameters $t_{H, \mathrm{Ge}}^{s s}$ and $t_{H, \mathrm{Ge}}^{s p_{y}}$ in the above equation corresponding to the matrix elements related to the $\mathrm{H}-\mathrm{Ge}$ bond. We adopt from the fitting procedure the numerical values $E_{H}^{s}=-2.54 \mathrm{eV}, t_{H, \mathrm{Ge}}^{s s}=V_{H, \mathrm{Ge}}^{s s}=-4.54 \mathrm{eV}$, and $t_{H, \mathrm{Ge}}^{s p_{y}}= \pm V_{H, \mathrm{Ge}}^{s p}$ with $V_{H, \mathrm{Ge}}^{s p}=0.5 \mathrm{eV}$ where $+(-)$ denotes the lower (upper) $\mathrm{H}-\mathrm{Ge}$ edge bonds. One can diagonalize the corresponding TB Hamiltonian, Eq. (16), in order to obtain the energy spectrum. By applying biaxial tensile strain we found that the band gap of the nanoribbon gradually decreases and eventually the metallic edge states protected by TRS appear for a strain value where a band inversion takes place in the TB energy spectrum of bulk monolayer $\mathrm{GeCH}_{3}$. The numerically calculated energy bands of $\mathrm{z}-\mathrm{GeCH}_{3}-\mathrm{NR}$ with $N=40$ in the presence of $9 \%, 11 \%$, and $13 \%$ biaxial tensile strain are shown
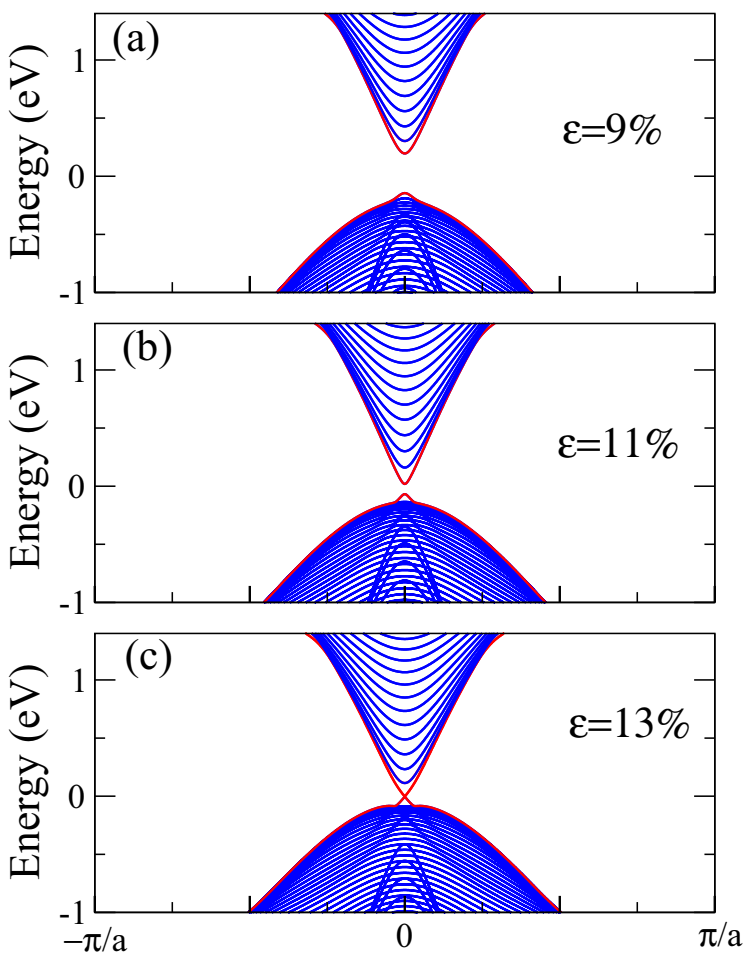

FIG. 10. The $1 \mathrm{D}$ energy bands of $\mathrm{z}-\mathrm{GeCH}_{3}-\mathrm{NR}$ for $N=40$ in the presence of (a) $9 \%$, (b) 11\%, and (c) $13 \%$ biaxial tensile strain.

in Figs. 10(a), 10(b) and 10(c), respectively. This demonstrates a topological phase transition from the NI to the QSH phase in the electronic properties of monolayer $\mathrm{GeCH}_{3}$.

\section{CONCLUSIONS}

To conclude, we have proposed an effective TB model with and without SOC for monolayer $\mathrm{GeCH}_{3}$ including $s$, $p_{x}$, and $p_{y}$ orbitals per atomic site. Our model reproduces the low-energy spectrum of monolayer $\mathrm{GeCH}_{3}$ in excellent agreement with $a b$ initio results. It also predicts accurately the evolution of the band gap in the presence of biaxial tensile strain. By including the SOC, this band gap manipulation leads to a band inversion in the electronic properties of monolayer $\mathrm{GeCH}_{3}$, giving rise to a topological phase transition from NI to QSH. Our model predicts that this phase transition takes place for $11.6 \%$ biaxial tensile strain as verified by the $\mathbb{Z}_{2}$ formalism. The topologically protected global bulk gap at a strain of $12.8 \%$ is $115 \mathrm{meV}$, which is much larger than the thermal energy at room temperature and makes monolayer $\mathrm{GeCH}_{3}$ a promising candidate for future applications. We also showed the emergence of topologically protected edge states in a typical $\mathrm{z}-\mathrm{GeCH}_{3}-\mathrm{NR}$ in the presence of biaxial strain larger than $11.6 \%$. This is an additional confirmation of the existence of the TI phase in the electronic properties of monolayer $\mathrm{GeCH}_{3}$.

M.R. and E.T.S. contributed equally to this work.
[1] C. L. Kane and E. J. Mele, Phys. Rev. Lett. 95, 226801 (2005).

[2] M. Z. Hasan and C. L. Kane, Rev. Mod. Phys. 82, 3045 (2010).
[3] X.-L. Qi and S.-C. Zhang, Rev. Mod. Phys. 83, 1057 (2011).

[4] J. E. Moore, Nature (London) 464, 194 (2010). 
[5] L. Fu, C. L. Kane, and E. J. Mele, Phys. Rev. Lett. 98, 106803 (2007).

[6] B. A. Bernevig, T. L. Hughes, and S.-C. Zhang, Science 314, 1757 (2006).

[7] A. J. Mannix, B. Kiraly, M. C. Hersam, and N. P. Guisinger, Nat. Rev. Chem. 1, 0014 (2017).

[8] A. C. Neto, F. Guinea, N. M. Peres, K. S. Novoselov, and A. K. Geim, Rev. Mod. Phys. 81, 109 (2009).

[9] M. Katsnelson, Graphene: Carbon in Two Dimensions (Cambridge University Press, Cambridge, 2012).

[10] Y. Yao, F. Ye, X.-L. Qi, S.-C. Zhang, and Z. Fang, Phys. Rev. B 75, 041401 (2007).

[11] D. Huertas-Hernando, F. Guinea, and A. Brataas, Phys. Rev. B 74, 155426 (2006).

[12] C. Si, J. Liu, Y. Xu, J. Wu, B.-L. Gu, and W. Duan, Phys. Rev. B 89, 115429 (2014).

[13] Z. Qiao, W.-K. Tse, H. Jiang, Y. Yao, and Q. Niu, Phys. Rev. Lett. 107, 256801 (2011).

[14] H. Pan, Z. Li, C.-C. Liu, G. Zhu, Z. Qiao, and Y. Yao, Phys. Rev. Lett. 112, 106802 (2014).

[15] Y. Ren, Z. Qiao, and Q. Niu, Rep. Prog. Phys. 79, 066501 (2016).

[16] D. C. Elias, R. R. Nair, T. Mohiuddin, S. Morozov, P. Blake, M. Halsall, A. Ferrari, D. Boukhvalov, M. Katsnelson, A. Geim, and K. S. Novoselov, Science 323, 610 (2009).

[17] S. Jiang, S. Butler, E. Bianco, O. D. Restrepo, W. Windl, and J. E. Goldberger, Nat. Commun. 5, 3389 (2014).
[18] S. Jiang, M. Q. Arguilla, N. D. Cultrara, and J. E. Goldberger, Acc. Chem. Res. 48, 144 (2014).

[19] E. Bianco, S. Butler, S. Jiang, O. D. Restrepo, W. Windl, and J. E. Goldberger, ACS Nano 7, 4414 (2013).

[20] Y. Ma, Y. Dai, W. Wei, B. Huang, and M.-H. Whangbo, Sci. Rep. 4, 7297 (2014).

[21] Y. Jing, X. Zhang, D. Wu, X. Zhao, and Z. Zhou, J. Phys. Chem. Lett. 6, 4252 (2015).

[22] S. Ma, F. Li, and C. Jiang, J. Elect. Mat. 45, 5412 (2016).

[23] T. P. Kaloni and U. Schwingenschlögl, Chem. Phys. Lett. 583, 137 (2013).

[24] See https://www.nature.com/article-assets/npg/srep/2014/ 141203/srep07297/extref/srep07297-s1.doc for HSE band structures.

[25] J. Heyd, G. E. Scuseria, and M. Ernzerhof, J. Chem. Phys. 118 8207 (2003).

[26] G. Bir and G. Pikus, Symmetry and Strain-induced Effects in Semiconductors (Wiley, New York, 1974).

[27] W. A. Harrison, Elementary Electronic Structure (World Scientific, Singapore/London, 1999).

[28] L. Li, X. Zhang, X. Chen, and M. Zhao, Nano Lett. 15, 1296 (2015).

[29] M. Zhao, X. Chen, L. Li, and X. Zhang, Sci. Rep. 5, 8441 (2015).

[30] L. Fu and C. L. Kane, Phys. Rev. B 74, 195312 (2006).

[31] T. Fukui and Y. Hatsugai, J. Phys. Soc. Jpn. 76, 053702 (2007).

[32] Y. Hatsugai, J. Phys. Soc. Jpn. 73, 2604 (2004). 